\title{
A HEM

\section{El uso de las tecnologías digitales en las escuelas: un estudio basado en los informes de políticas públicas brasileñas}

Use of digital technologies in school: a study based on the reports of Brazilian Public Policies

Uso de tecnologias digitais na escola: um estudo fundamentado nos relatórios de Políticas Públicas brasileiras

* Siderly do Carmo Dahle de Almeida

Professora doutora no Centro Universitário Internacional Uninter, Curitiba, Paraná, Brasil. siderly.c@gmail.com - http://orcid.org/0000-0002-2190-7213

** Alvaro Martins Fernandes Junior

Doutorando na Pontifícia Universidade Católica de São Paulo, São Paulo, São Paulo, Brasil. alvarojunior777@gmail.com - https://orcid.org/0000-0003-0726-1177

*** Viviane Marques Goi

Doutoranda na Pontificia Universidade Católica de São Paulo, Brasil vmgoi@terra.com.br - https://orcid.org/0000-0003-4491-9847

Recebido em 04 de fevereiro de 2019

Aprovado em 13 de junho de 2019

Publicado em 11 de setembro de 2019

\section{RESUMEN}

La educación se configura como protagonista en los programas de países que delinearon como meta valorar la calidad de vida de sus ciudadanos. Esta coyuntura apunta a la inminencia en lograr un retorno rápido, legítimo y efectivo para la interrupción de ciclos de pobreza y el establecimiento de objetivos que promuevan la libertad, la armonía y el progreso. Considerando el contexto de las Políticas Públicas en cuanto al uso de tecnologías en las escuelas, el objetivo de esta investigación fue percibir si la propuesta pedagógica de inclusión digital ya es una realidad nacional. Para ello, se realizó un estudio basado en cuatro documentos oficiales. Entre las cuestiones planteadas, se percibe que no hay mucha diferencia en cuanto al uso de TIC entre escuelas públicas y privadas, además, el celular es señalado como medio por el cual más se accede a internet y que cuando una institución enfatiza la cultura digital, asume la centralidad en la formación de alumnos más autónomos.

Palabras clave: Cultura digital en las escuelas; Inclusión digital en las escuelas; TIC y políticas públicas. 


\section{Aitloabão}

ISSN: 1984-6444 | http://dx.doi.org/10.5902/1984644436752

\section{ABSTRACT}

Education is a protagonist in the programs of countries that outlined the goal to value the life quality of its citizens. This situation points to the imminence of achieving a speedy, legitimate and effective return to the interruption of cycles of poverty and the establishment of objectives that promote freedom, harmony, and progress. Considering the context of public policies regarding the use of technologies in schools, the objective of this paper was to understand if the pedagogical proposal of digital inclusion is already a national reality. For this, a study was carried out based on 4 official documents. Among the issues raised is that there is not much difference regarding the use of ICTs between public and private schools, that the cell phone is pointed out as the medium through which the Internet is accessed the most and that when an institution emphasizes the digital culture, it assumes the centrality in the formation of more autonomous students.

Keywords: Digital Culture in Schools; Digital Inclusion in Schools; ICT and Public Policies.

\section{RESUMO}

A educação configura-se como protagonista nos programas de países que delinearam como meta valorizar a qualidade de vida de seus cidadãos. Esta conjuntura aponta para a iminência em se conseguir retorno célere, legítimo e efetivo para a interrupção de ciclos de pobreza e o estabelecimento de objetivos que promovam a liberdade, a harmonia e o progresso. Considerando-se o contexto das Políticas Públicas quanto ao uso de tecnologias nas escolas, o objetivo desta pesquisa foi perceber se a proposta pedagógica de inclusão digital já é uma realidade nacional. Para isso, realizou-se um estudo tendo por base 4 documentos oficiais. Dentre as questões levantadas, percebe-se que não há muita diferença quanto ao uso de TIC entre escolas públicas e privadas, além disso, o celular é apontado como meio pelo qual mais se acessa a internet e que quando uma instituição enfatiza a cultura digital, assume a centralidade na formação de alunos mais autônomos.

Palavras-chave: Cultura digital nas escolas; Inclusão digital nas escolas; TIC e políticas públicas.

\section{Introducción}

La sociedad pone en la educación la esperanza de que ésta venga a contribuir a la evolución y la prosperidad de la humanidad, considerando los preceptos de sostenibilidad y democracia. De este modo, es posible reducir el hambre, la 


\section{F HEM Eulloahá}

ISSN: 1984-6444 | http://dx.doi.org/10.5902/1984644436752

segregación social, la privación de oportunidades, la inseguridad en todos los ámbitos, problemas comunes que afligen y aniquilan poblaciones alrededor del mundo.

A partir de la década de los 90, la educación tomó lugar en la agenda de la comunidad científica, que se volvió a la repercusión de que la educación formal puede representar una gran contribución en cuanto al desarrollo de las naciones. Pactos fueron organizados, se establecieron compromisos y se definieron objetivos que pudieran garantizar la oportunidad de acceso a la escuela, considerando sus diferentes niveles, desde la Educación Infantil hasta la Educación Superior, así como formación profesional, técnica, permanente, sin olvidar el vínculo que se hace entre educación y mundo del trabajo y de la práctica social.

La Ley de Directrices y Bases de la Educación Nacional - LDBEN 9394/96 es un claro ejemplo de esta posición asumida por países en proceso de desarrollo, al tejer todo el documento basándose en su inspiración "en los principios de libertad y en los ideales de solidaridad humana" (BRASIL, 1996) ${ }^{1}$.

En este mismo período histórico, que se inicia en la década de los 90, se observa una efervescencia proveniente de las tecnologías digitales de información y de comunicación que pasan a interferir en los más diversos sectores de la sociedad: del modo como una noticia llega a su destino al modo con las instituciones comerciales venden sus productos. Cambios significativos son perceptibles en lo que se refiere al ocio, al sector de servicios, la industria y la educación, punto central de este estudio, cuyas instituciones pasan por modificaciones en sus estructuras.

Solamente después de veinte años de la promulgación de LDBEN en Brasil es que hay una alteración en que se consideran las tecnologías: La ley 13.415 de febrero de 2017, añade en su artículo 35 el término "y sus tecnologías" al referirse a las áreas del conocimiento: lenguajes, matemáticas y ciencias de la naturaleza (BRASIL, 2017), evidenciando la importancia de pensar en las áreas del conocimiento también bajo el enfoque de las tecnologías, aunque de forma no muy definida y clara.

El objetivo de esta investigación es percibir si la propuesta pedagógica de inclusión digital en las escuelas ya es una realidad nacional en Brasil. Para ello, se realizó un compilado de cuatro textos de los últimos tres años que abordan tal asunto. Son los siguientes documentos: 


\section{Autดaคูão}

ISSN: 1984-6444 | http://dx.doi.org/10.5902/1984644436752

- "TIC Educação 2016: pesquisa sobre o uso das tecnologias de informação e comunicação nas escolas brasileiras" (TIC Educación 2016: investigación sobre el uso de las tecnologías de información y comunicación en las escuelas brasileñas), producido por el Comité Gestor de Internet en Brasil (CGI Br, 2017);;

- "Marco Referencial Metodológico para a Medição do Acesso e Uso das Tecnologias de Informação e Comunicação (TIC) na Educação" (Marco referencial metodológico para la medición del acceso y uso de las tecnologías de la información y la comunicación (TIC) en la educación), producido por el Centro Regional de Estudios para el Desarrollo de la Sociedad de la Información (CETIC, 2016);

- "Pesquisa brasileira de mídia 2015: hábitos de consumo de mídia pela população brasileira" ("Investigación brasileña de medios de comunicación 2015: hábitos de consumo de medios por la población brasileña"), desarrollada por la Secretaría de Comunicación Social de la Presidencia de la República (BRASIL, 2014);

- "TIC Educação e desenvolvimento social na América Latina e o Caribe" (TIC Educación y desarrollo social en América Latina y el Caribe) un documento redactado por Enrique Hinostroza, director del Instituto de Informática Educativa de la Universidad de la Frontera de Chile (UNESCO, 2017).

La comparación entre los datos demostrados en estos cuatro documentos se basó en el alumno de la educación básica como unidad de análisis, excluyendo en ese contexto la educación infantil. Las categorías investigadas se refieren al perfil demográfico de esa población, al perfil específico relacionado al uso de las TIC en las escuelas y las actividades escolares, considerando las tecnologías usadas y el modo en que contribuyen con el proceso de enseñanza y aprendizaje.

El estudio se estructuró en cuatro partes así distribuidas: esta introducción aclarando contexto, objeto, objetivo y categorías de análisis; una sección que remonta las últimas tres décadas de la educación y el uso de tecnologías, fundamentándose 


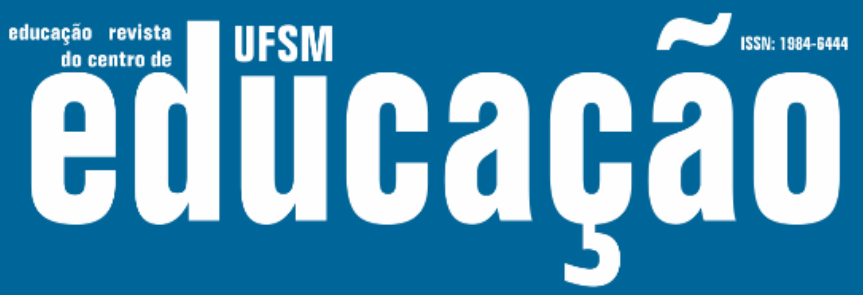

ISSN: 1984-6444 | http://dx.doi.org/10.5902/1984644436752

en autores como Levy (1999), Castells (1999, 2004), Galimberti (2006); una sección explicitando metodología, presentación y análisis de datos y, en una última parte, las consideraciones finales de la investigación.

\section{La integración de la tecnología a la educación: Breve historia}

En la década del 70 del siglo pasado las computadoras ya estaban en los centros de procesamiento de datos de las universidades brasileñas, y tenían como finalidad, prioritariamente, su uso administrativo, pero también era tímidamente utilizado para investigaciones específicas.

Las iniciativas referentes a la aplicación de tecnologías digitales de información y de comunicación en la educación básica surgieron a mediados de la década de 1980. En ellas se tomaba en consideración, de manera especial, el empeño en instituir Políticas Públicas que tendrían en cuenta la preparación de profesionales para actuación en nuevos escenarios que se delineaban en el mundo.

Aunque todavía rudimentaria, el Brasil tenía la Secretaría de Informática, la SEI (Secretaría Especial de Informática), órgano vinculado al Consejo Nacional de Seguridad y responsable por reglamentar, coordinar, supervisar y fomentar el desarrollo del sector. En 1983, esta secretaría instituyó la Comisión Especial № 11/83 - Informática en Educación, que tenía como objetivo proponer la orientación básica, los estándares de especificación técnica de equipamiento, la localización de los recursos y el monitoreo de la implementación de los centros piloto (MORAES, 1993). En 1984, se implantaron los primeros centros en la Universidad Estadual de Campinas, Universidad Federal de Minas Gerais, Universidad Federal de Pernambuco, Universidad Federal de Río de Janeiro y Universidad Federal de Rio Grande do Sul.

El establecimiento de políticas de inclusión digital se vuelve urgente y la educación se apunta como primordial en este escenario. Tales políticas pasan a finales del siglo pasado a ofrecer incentivo para el desarrollo de investigaciones en el área, así como programas de cualificación profesional y formación continuada de docentes y, además, inclusión de dispositivos tecnológicos en las escuelas, 


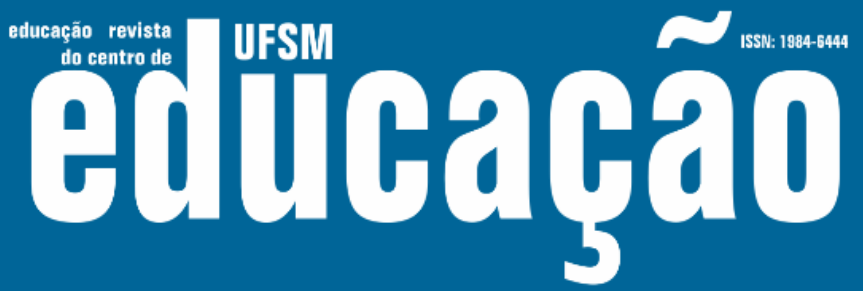

ISSN: 1984-6444 | http://dx.doi.org/10.5902/1984644436752

considerando la implantación de infraestructura y conexión a Internet. Se inicia un período de distintos desafíos a ser superados dentro y fuera de la escuela, entre éstos, la desigualdad social y la democratización del acceso a la información ya la cultura.

\section{Los procesos de aprendizaje mediados por la tecnología}

Para Castells, a partir de la década del 70 se iniciaron tres movimientos históricos autosuficientes que confluyeron hacia la "génesis de un nuevo mundo" (1999, p. 412). En principio hubo una crisis del capitalismo y un declive del Estado y su consecutiva reestructuración, nombrada por el autor como "capitalismo informacional": El principio se basa en la "producción por el lucro y para la apropiación privada de los beneficios basados en los derechos de propiedad" (p. 413). Sin embargo, tal reestructuración tiene como objetivo el desarrollo y la expansión tecnológica, la acumulación de información y, consecuentemente, la complejidad en los procesos de producción, desarrollo y diseminación del conocimiento en lugar de la potenciación de la producción de bienes consumibles.

El segundo movimiento primordial para el nuevo perfil de la sociedad contemporánea, se asocia a la culminación de la organización de distintos movimientos sociales y culturales, que instituyen el escenario para rupturas en los cimientos de la sociedad. Castells (1999) se refiere a temas que involucra las cuestiones de género, los cuidados con la vida en el planeta, la defensa de los derechos humanos, entre otros. Estos movimientos surgen como respuesta en oposición a la autoridad, a las desigualdades sociales, a los valores pregonados por el tradicionalismo religioso y buscaba exaltar la libertad de cada individuo. Las tecnologías, según el autor, determinaron las bases intelectuales para un mundo nuevo al que él denomina "sociedad en red" (CASTELLS, 1999).

Al mismo tempo de los dos movimientos, intercurrió un tercero, que es el de transformación de la sociedad y que se encuentra estrechamente relacionado con la revolución causada por las tecnologías digitales de la información, que funciona recomponiendo los cimientos materiales de la sociedad, a través del informacionalismo: atributo de la "forma de la organización social en que la 


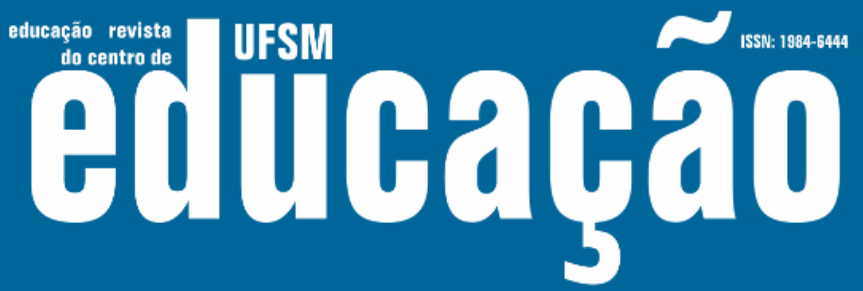

ISSN: 1984-6444 | http://dx.doi.org/10.5902/1984644436752

generación, el procesamiento y la transmisión de la información se convierten en fuentes fundamentales de productividad y poder [...] "(CASTELLS, 2006: 65).

Se cunan aquí las expresiones "sociedad informacional" y "economía informacional" empleados por Castells para diferenciar las transformaciones sociales, culturales y económicas contemporáneas y aún elevar las atribuciones de la información y del conocimiento en este contexto.

Galimberti (2006) evidencia que en esta "sociedad tecnológica" existe una transición en las condiciones que reflejan la relación del hombre con la naturaleza y con él mismo. El cambio paradigmático de lo que era la Sociedad Industrial para la Sociedad del Conocimiento (o Tecnológica) señala cambios de concepción productiva de cantidad para calidad. La técnica surge entonces como condición sine-qua-non para decodificar el mundo confiriéndole conceptos que su propio uso técnico le permite.

Pierre Lévy apunta que la tecnología traduce la habilidad que la humanidad tiene para dar impulso a su dominio de las tecnologías, a través de sus instituciones sociales. La obra de este autor, en general, es importante para la comprensión del uso que el hombre hace de la tecnología, teniendo en vista su formación orientada a la ciencia de la información y de la comunicación. En concreto, en su libro "Cibercultura", Lévy define tres principios esenciales que orientaron el crecimiento inicial del ciberespacio: "la interconexión, la creación de comunidades virtuales y la inteligencia colectiva" (LÉVY, 1999, p. 127). En este sentido, el conocimiento generado y compartido en el ciberespacio parte del principio de la reciprocidad, o sea, lo que es útil a una persona puede ser útil para muchas otras personas. Este nuevo ciclo de conocimiento tecnológico permitió a los usuarios interactuar con los instrumentos aprendiendo, apropiándose de sus mecanismos y redefiniendo su forma de utilizarlo.

En busca de propiciar la articulación entre distintas áreas de saber, especialmente en las dos primeras décadas del siglo XXI, las instituciones de enseñanza procuraron utilizar el ordenador como una herramienta de aprendizaje en el desarrollo de proyectos. En esta última perspectiva, el ordenador es utilizado por el alumno para configurar la solución de un problema o implantar un proyecto, creando sus propios modelos mentales. 


\section{ح

ISSN: 1984-6444 | http://dx.doi.org/10.5902/1984644436752

Almeida (2007) analiza que

Si usted piensa en la calidad de la educación, el ordenador puede dar una contribución realmente grande. Pero no es él solo. Él tiene que componer un proyecto político-pedagógico de la escuela. El ordenador sólo puede ayudar a una escuela que tiene un buen proyecto pedagógico. Si la escuela no tiene proyecto, o tiene un proyecto equivocado, mal elaborado, fragmentado, el ordenador empeora ese proyecto, ese proyecto se vuelve peor aún. En realidad, el ordenador es un optimizador de lo bueno o del pésimo. Con él, el proyecto de la escuela puede quedar mucho peor, si el proyecto es malo (ALMEIDA, 2007, p. 120) ${ }^{2}$

Así, se siguieron programas de gobierno provenientes de las esferas Federal, Estadual y Municipal. Además, surgió el interés de las instituciones privadas de enseñanza de insertar en sus currículos tecnologías digitales de información y comunicación, por un lado atrayendo alumnos en todos los niveles de enseñanza y, por otro, preocupándose por la formación del profesor para este nuevo contexto.

De acuerdo con Almeida y Valente (2016)

Se deben ofrecer oportunidades a los profesores para la apropiación pedagógica de los medios y las TIC, de modo que puedan integrarlas a los procesos de enseñanza, aprendizaje, desarrollo del currículo, evaluación e investigación sobre la propia práctica, utilizándolas para atender las necesidades de los estudiantes. Con tales competencias, los profesores se vuelven capaces de analizar por qué, para qué, con qué, cómo y cuándo integrar ese conocimiento a la práctica pedagógica. (ALMEIDA; VALENTE, 2016, p. 37) ${ }^{3}$

Almeida y Valente subrayan que es sustancial proporcionar que todos los involucrados en el proceso educativo - gestores, profesores, alumnos, equipo pedagógico, miembros del consejo de la escuela y de la comunidad - se movilicen y elaboren un plan que articule "infraestructura, recursos físicos, financieros y de infraestructura, tiempo y espacio de la escuela "(2016, p.37), propiciando y encadenando el diálogo entre todos y proporcionando oportunidades para el acceso y el uso de las tecnologías en el ambiente escolar.

Los profesores que actúan con educación básica comprenden que aliar tecnología al currículo permite diversas formas de interacción: profesor - alumno, alumno - profesor, alumno - alumno; ofrece informaciones variadas, superficiales o profundas, sobre todos los asuntos; que hace la clase más agradable y con mayor interés por parte de los alumnos; expande el aprendizaje más allá del aula y de la 


\section{Wusm oltuaráo

ISSN: 1984-6444 | http://dx.doi.org/10.5902/1984644436752

escuela; favorece la socialización de los saberes; ayuda en la inclusión digital tanto de alumnos como de los propios profesores.

Considerando que estas discusiones figuran como objeto de estudio y de trabajo de muchos investigadores alrededor del mundo, especialmente en los últimos treinta años, este estudio se ha vuelto a medir el acceso de la población a las tecnologías digitales, para alcanzar el objetivo propuesto, que es investigar si, efectivamente, las tecnologías digitales de información y comunicación son utilizadas en la educación brasileña.

\section{Metodología, presentación y análisis de datos}

Como se anunció en la introducción de este artículo, este estudio se respaldó en cuatro documentos:

- “TIC Educação 2016" (TIC Educación 2016); O "Marco Referencial Metodológico para a Medição do Acesso e Uso das Tecnologias de Informação e Comunicação (TIC) na Educação" (Marco referencial metodológico para la medición del acceso y uso de las tecnologías de la información y la comunicación (TIC) en la educación), a "Pesquisa brasileira de mídia 2015: hábitos de consumo de mídia pela população brasileira" ("Investigación brasileña de medios de comunicación 2015: hábitos de consumo de medios por la población brasileña"), desarrollada por la Secretaría de Comunicación Social de la Presidencia de la República (BRASIL, 2014) y "TIC Educação e desenvolvimento social na América Latina e o Caribe" (TIC Educación y desarrollo social en América Latina y el Caribe) un documento redactado por Enrique Hinostroza, director del Instituto de Informática Educativa de la Universidad de la Frontera de Chile (UNESCO, 2017).

La unidad de análisis de este estudio fue el alumno que frecuenta la educación básica. Las categorías elegidas para mayor profundización son el perfil demográfico de esa población, el perfil específico relacionado al uso de las TIC en las escuelas y 


\section{Nusm Eutbapga}

ISSN: 1984-6444 | http://dx.doi.org/10.5902/1984644436752

las actividades escolares, considerando las tecnologías usadas y cómo los profesores hacen uso de las mismas.

Considerando la investigación TIC Educación 2016, se utilizó la Parte 2, que presenta el informe metodológico y el análisis de los resultados de las TIC Educación 2016. Con respecto al Marco Referencial, producido por el CETIC, se analizaron los textos que se refieren a los dilemas entre las prácticas instructivas y la cultura digital en la integración entre currículo y TIC. El enfoque del tercer documento, la Encuesta Brasilera de Medios de 2015, fue el capítulo 3 - Internet - que, luego en su introducción, alerta que Internet fue apuntada por el $42 \%$ de los brasileños $\left(1^{\circ}+2^{\circ}+3^{\circ}\right.$ lugares) como el medio de comunicación más utilizado, quedando atrás de la televisión (93\%) y, por una pequeña diferencia, de la radio (46\%) (BRASIL, 2014). En el último documento que compone el estudio se hizo una lectura de todo el texto por tratarse de un documento breve, pero que merece atención por presentar datos de América Latina y el Caribe a lo largo del año 2016.

\section{TIC educación 2015: investigación sobre el uso de las tecnologías de información y comunicación en las escuelas brasileñas}

La investigación TIC Educación tuvo por objetivo reconocer el acceso, la utilización y la apropiación de las TIC en las escuelas públicas y privadas del país, tanto en lo que concierne a la práctica pedagógica en cuanto a la gestión escolar, investigando las escuelas, los directores, los coordinadores pedagógicos, profesores y alumnos. Como anteriormente explicitado, específicamente en este estudio, la unidad de análisis elegida fue el alumno y, de esa forma, la mirada de los investigadores siguió en esta dirección.

Es importante resaltar que en el instrumento de recolección de datos de TIC Educación, en lo que se refiere a los alumnos "se excluyeron las cuestiones sobre: uso de red WiFi de la escuela aunque restringido, habilidades generales, lugar de realización de actividades escolares con uso de Internet. Se incluyó una pregunta sobre el uso del teléfono celular en actividades escolares con orientación del profesor "(CGI Br, 2017). La unidad de análisis, por lo tanto, recae sobre 1.087 Instituciones de 


\section{Eilthapão}

ISSN: 1984-6444 | http://dx.doi.org/10.5902/1984644436752

Enseñanza que respondieron al cuestionario de alumnos, sumando 11.069 alumnos respondedores.

Desde el punto de vista de los alumnos sobre el uso de tecnologías digitales que sus profesores hacen, se demuestra que están bien satisfechos, no siendo señaladas diferencias entre Instituciones públicas y privadas, sino entre los niveles de enseñanza y edad de los alumnos, según apunta el gráfico 1.

Gráfico 1 - Alumnos, por percepción sobre posibles impactos de las tic (2016) ${ }^{4}$

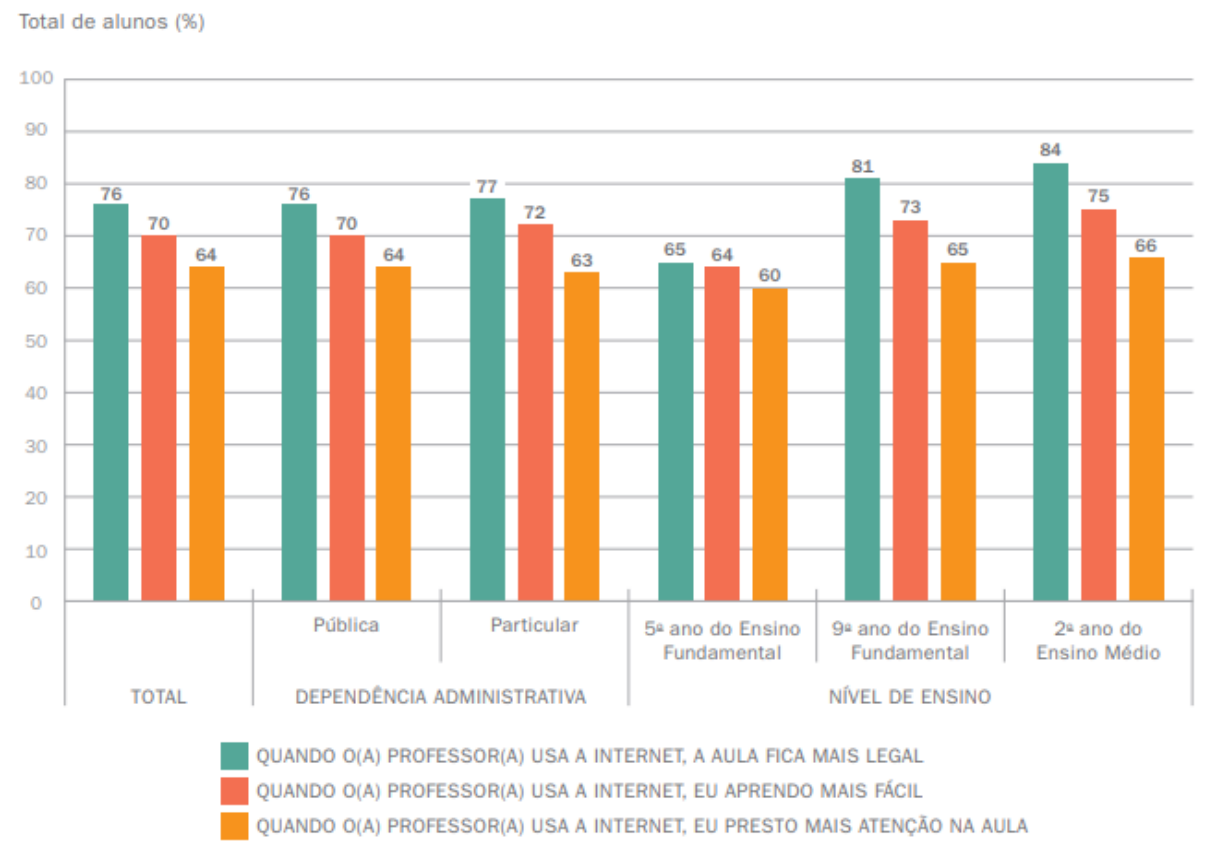

Fonte: CGI Br, 2017.

Estos datos evidencian que hay una gran similitud entre las respuestas dadas por alumnos de escuelas públicas y privadas sobre la motivación y el aprendizaje que proporciona Internet, independientemente del nivel en que los alumnos estudian. Sin embargo, del quinto año de la enseñanza primaria hasta al final de la enseñanza secundaria se observa una curva creciente en la preferencia por el uso de tecnologías, o sea, cuanto mayor la edad, mayor la conexión.

Otro dato importante se refiere a la ubicuidad que, según Almeida y Valente (2016), tiene que ver con el uso de medios y tecnologías digitales, independientemente del tiempo y del espacio en que el individuo esté. Se refiere a los 


\title{
Tilusm

ISSN: 1984-6444 | http://dx.doi.org/10.5902/1984644436752

dispositivos móviles ya los servicios que quedan en las nubes y permiten acceso instantáneo e interacción entre las personas que se encuentran temporal y espacialmente distantes. En vista del contexto educativo, el teléfono móvil fue apuntado por profesores y alumnos como el medio por el cual más accede a Internet, sea para verificar las redes sociales, como para la realización de tareas escolares. Según datos de la investigación,

\begin{abstract}
El teléfono móvil fue el principal dispositivo para acceso a Internet al $77 \%$ de los alumnos usuarios de la red, siendo que en 2015 esa proporción era del $73 \%$. Al mismo tiempo, el acceso a Internet prioritariamente por otros equipos, como computadoras de escritorio $(9 \%)$ y portátiles $(6 \%)$ presenta tendencia de reducción (CGI Br, 2017).
\end{abstract}

En cuanto al acceso a internet por el celular, otro dato interesante es que de este total de alumnos, el $21 \%$ está en escuelas públicas y el $5 \%$ en escuelas privadas. Esto ocurre porque los alumnos de escuelas privadas utilizan otros medios de acceso y, con la popularización del celular, lo mismo se ha convertido en el principal dispositivo entre los diversos segmentos de la población, incluso los de menor renta.

Es importante verificar que, a pesar de haber una gran parte de la población con posibilidad de acceso a internet, todavía hay un porcentaje de alumnos desconectados: "En la región Norte el 29\%, en el Nordeste (18\%), Sur (14\%), CentroOeste (12\%) y Sudeste (11\%) "(CGI Br, 2017, p. 110). En ese sentido hay, aún, mucho que hacer en la búsqueda de disminuir la exclusión digital en Brasil.

Según los datos de la investigación, en 2016 todavía había un número inmenso de escuelas que prohibían el uso del celular en el aula: el 5\% de alumnos de las escuelas públicas y el $9 \%$ de escuelas privadas dijeron que era permitido utilizar el celular en el aula (CGI Br, 2017). A esta información, se suma el número limitado de escuelas con redes de wifi que permiten acceso a sus estudiantes, el $61 \%$ de las escuelas no liberan las contraseñas de acceso a sus alumnos. 


\section{F HEM Eulloahá}

ISSN: 1984-6444 | http://dx.doi.org/10.5902/1984644436752

\section{Marco referencial metodológico para la medición del acceso y uso de las tecnologías de información y comunicación (TIC) en la educación}

Este documento, elaborado en 2016, tuvo por objetivo producir un marco referencial que determinara dimensiones e indicadores oportunos para presentar respuestas referentes al contexto regional de América Latina, más específicamente de Brasil, disponiendo de la "producción sistemática de estadísticas comparables internacionalmente y al mismo tiempo relevantes para las políticas públicas específicas de cada país "(CETIC, 2016).

Se resalta en el texto que la Agenda 2030 para el Desarrollo Sostenible de las Organizaciones de las Naciones Unidas (ONU) presenta actitudes y criterios transformadores para la prosperidad de los ciudadanos y del planeta como un todo, para vencer "el desafío de promover, de forma equilibrada, las tres dimensiones del desarrollo sostenible a nivel global: crecimiento económico, inclusión social y sostenibilidad ambiental "(CETIC, 2016). El objetivo 4 de esta agenda exalta la educación de calidad de manera a propiciar educación inclusiva y equitativa $y$, además, oportunizar el aprendizaje a lo largo de la vida para todos. Estos desafíos, de acuerdo con el informe, pueden ser enfrentados con la adopción de las tecnologías digitales como fuente de acceso al conocimiento y como herramienta pedagógica.

El texto aclara que frente a las transformaciones inherentes a las prácticas sociales estrechamente influenciadas por la cultura digital, la educación se ve frente a dilemas que presentan la falsa noción de oposición entre dos distintas visiones de currículo, siendo preciso resaltar las convergencias entre "las prácticas sociales de la cultura digital, las políticas públicas y las intenciones educativas "(CETIC, 2016). De esta forma, la institución de enseñanza que se involucra con la cultura digital, estableciendo los necesarios diálogos con la misma, asume la centralidad en la formación del alumno autónomo, dirigiéndolo para que sepa argumentar y defender sus ideas, trabajar en grupo con respecto a las distintas ideas de sus colegas, actuar de forma crítica, activa, creativa y cuestionadora.

La idea aquí es que los alumnos aprenden con los profesores, así como los profesores aprenden con los alumnos. Jesus y Tasson explicitan que 


\section{U Lism

ISSN: 1984-6444 | http://dx.doi.org/10.5902/1984644436752

En esta relación dialógica (profesor-alumno) hay producción de conocimientos profesionales, a partir de la observación de que no todas las prácticas e intervenciones planificadas son eficaces, pues, a veces, los niños redireccionan la forma de mirar, hacia algo que no había sido predicho. En este sentido, los niños actúan en la zona de desarrollo proximal del profesor, haciéndole pensar en otras formas de trabajar, con el fin de acoger los cuestionamientos de los niños para hacerlos sentirse parte del proceso (JESUS; TASSON, 2017, p. 237). ${ }^{5}$

El documento enfatiza que con el uso de las tecnologías digitales de información y comunicación, nuevas prácticas se diseminan en el espacio escolar:

- Uso de juegos digitales (juegos) y el desarrollo de nuevos juegos por los estudiantes;

- Participación en redes sociales y comunidades creadas en Internet en torno a un tema de interés;

- Uso, creación y uso compartido de Recursos Educativos Abiertos (REA) disponibles en la web;

- Producción de vídeos, animaciones y narrativas digitales sobre experiencias de los estudiantes;

- Uso y creación de aplicaciones sobre temas específicos del currículo escolar, tales como simulaciones, experimentos virtuales;

- Participación de los estudiantes en laboratorios virtuales de ciencias;

- Participación colectiva en la creación de ciudades virtuales y en la discusión de sus problemáticas;

- Creación de glosarios digitales sobre temas específicos (CETIC, 2016).

Entre los factores que favorecen la cultura digital de la escuela, la premisa es el acceso ininterrumpido a Internet, de forma pública y gratuita, a todos los pertenecientes al proceso educativo. El texto explicita que es esencial permitir la "disponibilidad continua, de calidad y de capacidad adecuada de la infraestructura tecnológica de la escuela, lo que debe considerar políticas efectivas de soporte y mantenimiento de la infraestructura TIC" (CETIC, 2016). 


\section{usm Eulloabुa}

ISSN: 1984-6444 | http://dx.doi.org/10.5902/1984644436752

\section{La investigación brasileña de medios de comunicación 2015: hábitos de consumo de medios por la población brasileña}

Esta investigación es el mayor levantamiento acerca de los hábitos de información de los ciudadanos brasileños y se refiere a lo que ocurrió en el transcurso del año 2014. Importa particularmente en este estudio verificar el uso de internet que apuntaba que en el período el $48 \%$ de la población hacía uso de la red. En cuanto al tiempo de conexión, se observó que los usuarios "se conectan, en promedio, 4h59 por día durante la semana y 4 h24 los fines de semana". El año anterior, el promedio era de $3 h 39$ y $3 h 43$.

El informe apunta que el $51 \%$ de las personas todavía no utilizaban Internet en 2014 y que entre los que utilizaban, el 67\% buscaba información y noticias en general, "de diversión y entretenimiento (67\%), de una forma de pasar el tiempo libre (38\%) y de estudio y aprendizaje (24\%) "(BRASIL, 2014, p. 49)

Con respecto al nivel de enseñanza, se verificó que el $72 \%$ de las personas con Enseñanza Superior accede a internet por aproximadamente $5 \mathrm{~h} 41$. Las personas que tienen sólo los años iniciales de la Enseñanza Fundamental utilizan mucho menos, sólo el $5 \%$ por 3h22. El $65 \%$ de los jóvenes de 16 a 25 años se conectan por 5 h51 y el $4 \%$ de los que tienen 65 años o más la utilizan por 2h53 (BRASIL, 2014).

En este año se empieza a haber equilibrio entre el acceso por medio de celular y por medio de computadoras de escritorio - $66 \%$ y $71 \%$, respectivamente.

\footnotetext{
Entre las redes sociales y los programas de intercambio de mensajes instantáneos más usados $\left(1+2^{\circ}+3^{\circ}\right.$ lugares), están Facebook $(83 \%)$, Whatsapp (58\%), Youtube (17\%), Instagram (12\% y Google + (8\%). Twitter, popular entre las elites políticas y formadores de opinión, fue mencionado sólo por el $5 \%$ de los entrevistados. En cuanto a los principales soportes de acceso a Internet $\left(1^{\circ}+2^{\circ}\right.$ lugares), los resultados muestran que la mayoría de los entrevistados (71\%) lo hacen vía computadora, seguido por el celular $(66 \%)$. También hay una pequeña parte $(7 \%)$ de los encuestados que utiliza tabletas para navegar por el mundo digital. (BRASIL, 2014) ${ }^{6}$
}

La investigación brasileña de medios no presenta con claridad los datos sobre el público que frecuenta la escuela, foco de este estudio, sin embargo, presenta datos importantes con relación al tiempo medio de conexión de los brasileños, además de 


\section{T WsM FutlaghaO}

ISSN: 1984-6444 | http://dx.doi.org/10.5902/1984644436752

las preferencias en cuanto a redes sociales y en cuanto al uso del uso, que ganaba por una pequeña diferencia del uso del celular cuando se habla de conexión a internet.

\section{TIC educación y desarrollo social en América latina y el caribe}

Este documento, redactado por el Dr. Enrique Hinostroza, director del Instituto de Informática Educativa de la Universidad de la Frontera de Chile para la Unesco, busca "plantear debates multidisciplinarios, proponer análisis basados en evidencia y formular propuestas de políticas públicas para consolidar una estrategia de desarrollo sostenible necesaria en América Latina y el Caribe, en el marco de la Agenda 2030 "(UNESCO, 2017, p.3).

Se explicita que para la democratización del acceso a la cultura digital, se hace necesario vencer dos desafíos: el acceso libre y gratuito a ordenadores conectados a internet y que esta conectividad sea de buena calidad. Otro punto planteado en el documento es que existe una gran disponibilidad de recursos educativos abiertos, pero no hay un estándar de calidad que contribuya a la selección, uso y diseminación de estos recursos.

Es importante recordar que las competencias digitales deben integrarse en el currículo escolar para contribuir a que las futuras generaciones aprovechen este potencial. Para ello, es imprescindible que los profesores incluyan el uso de la tecnología en su práctica pedagógica, buscando la construcción del conocimiento:

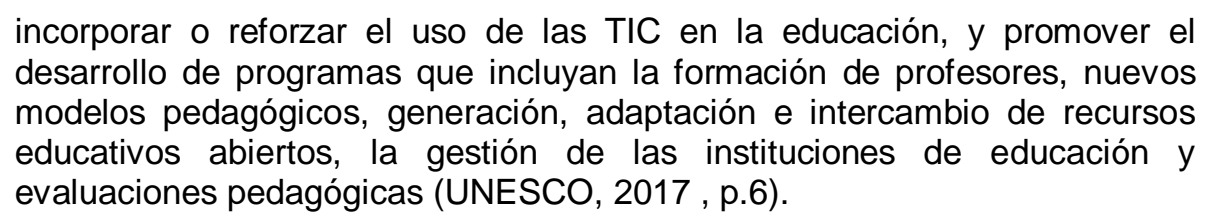

Brasil se encontraba en el 2016 con igual acceso a ordenadores y a Internet, en el rango del 50\%, lo que representa todavía muy poco y, por lo tanto, denota la necesidad de programas dirigidos tanto para la infraestructura y la posibilidad de adquisición de hardware y acceso a la red, en cuanto a la formación de las personas para el uso de estos equipos. 


\title{
T usm Allthapã

ISSN: 1984-6444 | http://dx.doi.org/10.5902/1984644436752

Este documento también pone de manifiesto la necesidad de

\begin{abstract}
garantizar a los estudiantes el acceso continuo (en la escuela y en casa) a ordenadores, principalmente al grupo de estudiantes de nivel socioeconómico inferior; ofrecer conexión a Internet a escuelas más distantes de los centros urbanos; y mejorar la calidad del acceso a Internet, es decir, garantizar la conexión de banda ancha con la velocidad necesaria para aprovechar los recursos y servicios disponibles. Además, en términos generales, se aconseja incentivar iniciativas para ofrecer conexión a Internet de calidad a los estudiantes y profesores fuera de la escuela (UNESCO, 2017, p. 14).
\end{abstract}

En todo caso, el documento alerta sobre el hecho de que la escuela no ha logrado realizar la integración de las tecnologías al currículo, sea por la falta de infraestructura, sea por la falta de formación pedagógica para tal uso. Además, alerta que la verdadera integración ocurre fuera del aula, evidenciando la necesidad de que los docentes se preparan con mayor rigor para el desarrollo de las competencias digitales, tan necesarias para la actuación en la escuela contemporánea.

El texto concluye que, concatenando los beneficios latentes de las tecnologías digitales, existen muchos desafíos que hay que afrontar y solucionar, como por ejemplo, la falta de acceso a esas tecnologías, así como la falta de preparación de la población para hacer uso de la misma.

\section{Consideraciones finales}

Esta investigación tuvo por objetivo percibir si la propuesta de inclusión digital ya es una realidad en las escuelas. Para ello, se realizó un compilado de cuatro textos oficiales de los últimos 4 años que abordan tal asunto. La confrontación entre las informaciones contenidas en estos cuatro documentos se orientó en el alumno de Educación básica como unidad de análisis. Las categorías investigadas se orientaron en el perfil demográfico de esa población, en el perfil específico relacionado al uso de las TIC en las escuelas y en las actividades escolares, considerando las tecnologías usadas y el modo como contribuyen con el proceso de enseñanza y de aprendizaje.

En el análisis de cada uno de los documentos es posible afirmar que el acceso, la utilización y la apropiación de las TIC en las escuelas públicas y privadas del país, en lo que concierne a la práctica pedagógica, se hace de modo muy similar, demostrando que los docentes están sí preocupados con tal inserción en el contexto 


\section{F HEM EUtrathâ}

ISSN: 1984-6444 | http://dx.doi.org/10.5902/1984644436752

escolar, siendo menos utilizado en los años iniciales de la Enseñanza Fundamental, exacerbándose el uso en los años finales y en la Enseñanza Media.

Se observó que los alumnos, tanto de escuelas públicas y de escuelas privadas, se sienten más motivados y dicen aprender mejor cuando hacen uso de Internet y, cuanto más viejos, mayor también el tiempo de conexión.

La investigación del CGI Br (2017) demostró que el teléfono móvil es el principal dispositivo de acceso a internet, tanto en la escuela, como en casa, por profesores y por alumnos. El documento enfatiza además que hay un gran porcentaje de alumnos desconectados, especialmente en la región Norte y en el Nordeste.

La investigación del CETIC (2016) evalúa que la educación se encuentra en un dilema cuando el asunto es tecnología y currículo. Es necesario percibir las convergencias entre ambas y fomentar su desarrollo. Así, no basta llevar la tecnología en las clases, es necesario concretar el currículo por medio de esta. De este modo, se hace posible formar al alumno autónomo, crítico, reflexivo, que sabe oír y posicionarse frente al conocimiento, sea presentado por el profesor o por sus colegas. Para poder integrar efectivamente la tecnología al currículo, el documento presenta una serie de prácticas que favorecen la introducción de una cultura digital en las escuelas.

En cuanto a los hábitos de los brasileños con respecto al uso de tecnologías de modo general, en 2014 , el $48 \%$ de la población estaba conectada y se ubicaba en promedio 5 horas al día en internet. Considerando el nivel de enseñanza de la población, se verificó que el $72 \%$ de las personas con Enseñanza Superior accede a internet por aproximadamente 5h41. Las personas que tienen sólo los años iniciales de la Enseñanza Fundamental utilizan mucho menos, sólo el 5\% por 3h22. El 65\% de los jóvenes de 16 a 25 años se conectan por 5h51. Una vez más se verifica que, cuanto más viejo, más tiempo conectado a la red.

La investigación contribuye a comprender que para la democratización efectiva del acceso a la cultura digital es necesario que haya acceso gratuito a los equipos, así como conectividad gratuita y de buena calidad. Otra cuestión importante es que se busca avanzar más en la calidad de los recursos educativos abiertos ofrecidos a la población. 


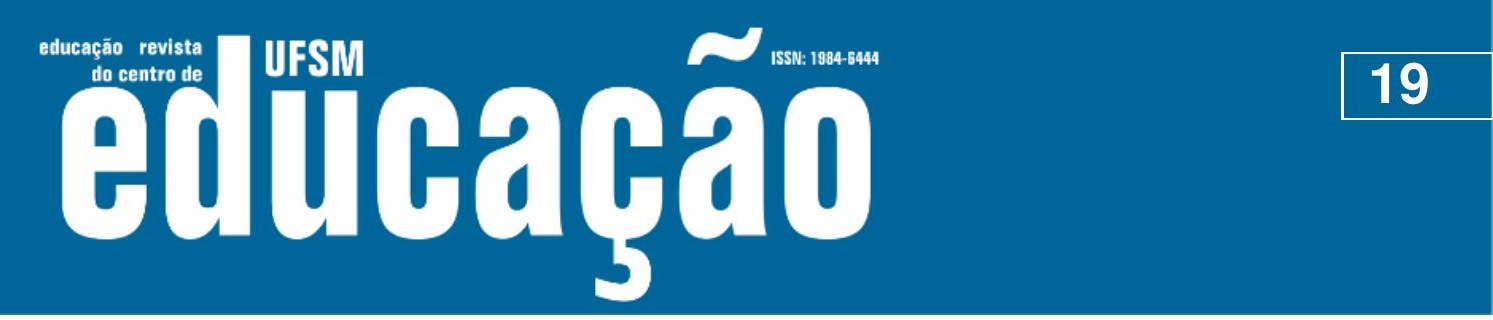

ISSN: 1984-6444 | http://dx.doi.org/10.5902/1984644436752

Superados los desafíos, las competencias digitales deben ser integradas al currículo escolar, calificando a las futuras generaciones para mejor vivir / convivir en la sociedad del conocimiento. También cabe enfatizar que la escuela, como espacio que no puede prescindir de estar a la vanguardia en lo que se refiere al uso de las tecnologías en la educación, necesita invertir en la calificación de su cuerpo docente.

\section{Referências}

ALMEIDA, Fernando José de. Moçambique está ficando perto: depoimento. Entrevistadora: Nilce da Silva. Revista Eletrônica Acolhendo a Alfabetização nos Países de Língua Portuguesa, São Paulo, USP, 2007. (Depoimento: Entrevista concedida ao Grupo Acolhendo Alunos em Situação de Eaxclusão Social, da Faculdade de Educação da Universidade de São Paulo e Pós-Graduação em Educação de Jovens e Adultos, da Faculdade de Educação da Universidade Eduardo Mondlane.

Disponível

em: https://www.revistas.usp.br/reaa/article/viewFile/11451/13219. Acesso em: 17 jun. 2018.

ALMEIDA, Maria Elizabeth Bianconcini.; VALENTE, José Armando. Políticas de tecnologia na Educação Brasileira: histórico, lições aprendidas e recomendações. In: Centro de Inovação para a educação brasileira, 2016. Disponível em: http://www.cieb.net.br/wp-content/uploads/2016/12/CIEB-Estudos-4-Politicas-deTecnologia-na-Educacao-Brasileira.pdf. Acesso em: 10 out. 2017.

BRASIL. Lei no 9.394, de 20 de dezembro de 1996. Estabelece as diretrizes e bases da educação nacional. Brasília: Ministério da Educação, 1996.

BRASIL. Lei 13.415, de 16 de fevereiro de 2017. Altera as Leis nos 9.394, de 20 de dezembro de 1996, que estabelece as diretrizes e bases da educação nacional, e 11.494, de 20 de junho 2007, que regulamenta o Fundo de Manutenção e Desenvolvimento da Educação Básica e de Valorização dos Profissionais da Educação, a Consolidação das Leis do Trabalho - CLT, aprovada pelo Decreto-Lei o 5.452, de 10 de maio de 1943, e o Decreto-Lei oㅡ 236, de 28 de fevereiro de 1967; revoga a Lei no11.161, de 5 de agosto de 2005; e institui a Política de Fomento à Implementação de Escolas de Ensino Médio em Tempo Integral.. Brasília, 2017.

BRASIL. Presidência da República. Secretaria de Comunicação Social. Pesquisa brasileira de mídia 2015: hábitos de consumo de mídia pela população brasileira. Brasília: Secom, 2014.

CASTELLS, Manuel. Cibercultura. São Paulo: Ed. 34, 1999. 


\section{T WsM Eutloabुa}

ISSN: 1984-6444 | http://dx.doi.org/10.5902/1984644436752

CASTELLS, Manuel. A sociedade em rede. 8. ed. rev. e ampl. São Paulo: Paz e Terra, 2006.

CETIC. Marco Referencial Metodológico para a Medição do Acesso e Uso das Tecnologias de Informação e Comunicação (TIC) na Educação. In: Centro Regional de Estudos para o desenvolvimento da Sociedade da Informação - Brasil. São Paulo: Unesco, 2016.

CGI Br - COMITÊ GESTOR DA INTERNET NO BRASIL. Pesquisa sobre o uso das tecnologias de informação e comunicação nas escolas brasileiras: TIC educação 2015 [livro eletrônico] = Survey on the use of information and communication technologies in brazilian schools: ICT in education 2015. São Paulo: Comitê Gestor da Internet no Brasil, 2016.

GALIMBERTI, Umberto. Psiche e techne, o homem na idade da técnica. São Paulo: Paulus, 2006.

JESUS, Deise Cristina Carvalho de; TASSON, Elvira Cristina Martins. Escritas de mim: narrativas e a autoformação docente. EccoS - Rev. Cient., São Paulo, n. 44, p. 225-240, set./dez. 2017.

LÉVY, Pierre. Cibercultura. São Paulo: Ed. 34, 1999.

MORAES, Maria Cândida. Informática Educativa no Brasil: um pouco de História. Em Aberto, Brasília, ano 12, n. 57, jan./mar. 1993. Disponível em http://emaberto.inep.gov.br/index.php/emaberto/article/view/1877/1848. Acesso em: 17 jun. 2018.

UNESCO. TIC Educação e desenvolvimento social na América Latina e o Caribe. Montevideu: Unesco, 2017.

\section{Correspondência}

Siderly do Carmo Dahle de Almeida - Centro Universitário Internacional Uninter Rodovia Curitiba - Ponta Grossa Br-277, 891. CEP 81020-490, Mossunguê, Curitiba, Paraná, Brasil.

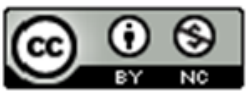

This work is licensed under a Creative Commons Attribution-NonCommercial 4.0 International (CC BY-NC 4.0) 


\section{N

ISSN: 1984-6444 | http://dx.doi.org/10.5902/1984644436752

\section{Notas}

\footnotetext{
${ }^{1}$ En el original es: "nos princípios de liberdade e nos ideais de solidariedade humana” (BRASIL, 1996).

${ }^{2}$ En el original es: "Se você pensa na qualidade da educação, o computador pode dar uma contribuição realmente grande. Mas não é ele sozinho. Ele tem que compor um projeto político-pedagógico da escola. O computador só pode ajudar uma escola que tem um projeto pedagógico bom. Se a escola não tiver projeto, ou tiver um projeto equivocado, mal elaborado, fragmentado, o computador piora esse projeto, esse projeto fica pior ainda. Na verdade, o computador é um otimizador do bom ou do péssimo. Com ele, o projeto da escola pode ficar muito pior, se o projeto for ruim" (ALMEIDA, 2007, p. 120)
}

${ }^{3}$ Em el original es: "Devem ser oferecidas oportunidades aos professores para a apropriação pedagógica das mídias e TIC, de modo que eles possam integrá-las aos processos de ensino, aprendizagem, desenvolvimento do currículo, avaliação e pesquisa sobre a própria prática, utilizando-as para atender às necessidades dos alunos. Com tais competências, os professores se tornam capazes de analisar por quê, para quê, com o quê, como e quando integrar esse conhecimento à prática pedagógica" (ALMEIDA; VALENTE, 2016, p. 37)

${ }^{4}$ Traducción del gráfico: En la parte superior: total de alumnos. Debajo de las barras: publica/particular/ quinto año de enseñanza primaria/Nono año de enseñanza primaria/ Segundo año de enseñanza secundaria. En los cuadrados: Azul: cuando el (la) profesor (a) usa la internet la clase queda más agradable. Rojo: cuando el (a) profesor (a) usa la internet aprendo más fácil. Naranja: cuando el (a) profesor (a) usa internet yo presto más atención en la clase.

${ }^{5}$ En el original es: "Nessa relação dialógica (professor-aluno) há produção de conhecimentos profissionais, a partir da observação de que nem todas as práticas e ou intervenções planejadas são eficazes, pois, às vezes, as crianças redirecionam a forma de olhar, para algo que não havia sido previsto. Nesse sentido, as crianças atuam na zona de desenvolvimento proximal do professor, fazendo-o pensar em outras formas de se trabalhar, visando acolher os questionamentos das crianças para fazê-las se sentirem parte do processo" (JESUS; TASSON, 2017, p. 237).

${ }^{6}$ Em el original: "Entre as redes sociais e os programas de trocas de mensagens instantâneas mais usadas $\left(1^{\circ}+2^{\circ}\right.$ $+3^{\circ}$ lugares), estão o Facebook (83\%), o Whatsapp (58\%), o Youtube (17\%), o Instagram (12\%) e o Google+ (8\%). O Twitter, popular entre as elites políticas e formadores de opinião, foi mencionado apenas por $5 \%$ dos entrevistados. Em relação aos principais suportes de acesso à internet $\left(1^{\circ}+2^{\circ}\right.$ lugares $)$, os resultados mostram que a maioria dos entrevistados $(71 \%)$ o fazem via computador, seguido pelo celular $(66 \%)$. Há ainda uma pequena parcela $(7 \%)$ dos pesquisados que utiliza tablets para navegar pelo mundo digital". (BRASIL, 2014) 